\title{
EL PAPEL DE LA CONVIVENCIA ESCOLAR EN LA FORMACIÓN INICIAL DEL PROFESORADO DE EDUCACIÓN INFANTIL Y PRIMARIA
}

\author{
The role of school coexistence in the initial training \\ of teachers in early childhood and primary education
}

Carlos MONGE-LÓPEZ* y Patricia GÓMEZ-HERNÁNDEZ***

* Universidad Complutense de Madrid. España.

camonge@ucm.es

bttps://orcid.org/0000-0002-7761-6087

* Centro Universitario Internacional de Madrid (centro adscrito a la Universidad de Alcalá). España.

patricia.gomez@cunimad.com

https://orcid.org/0000-0001-9163-7637

Fecha de recepción: 03/07/2020

Fecha de aceptación: 10/09/2020

Fecha de publicación en línea: 01/11/2020

Cómo citar este artículo: Monge-López, C. y Gómez-Hernández, P. (2021). El papel de la convivencia escolar en la formación inicial del profesorado de educación infantil y primaria. Teoría de la Educación. Revista Interuniversitaria, 33(1), 197-220. https://doi. org/10.14201/teri.23580

RESUMEN

La convivencia en los centros educativos supone un tema de interés en cuanto que condiciona sustancialmente los tipos y calidad de los aprendizajes. Pero la gestión de la convivencia escolar no es una cuestión fácil de abordar y requiere, al menos, una adecuada formación del profesorado. Por ello, el principal objetivo de esta investigación fue analizar qué contenidos, objetivos, competencias y recursos se están trabajando en la formación inicial del profesorado de Educación Infantil y Educación Primaria. 

DE EDUCACIÓN INFANTIL Y PRIMARIA

Para ello se analizaron las guías docentes (2019-2020) de las Facultades de Educación españolas posicionadas en el Shanghai Ranking (2.224 guías analizadas). Las cuestiones para analizar giraron en torno a: (1) la conceptualización y dimensionalidad de la convivencia, (2) la configuración y tipologías de conflictos escolares y (3) estructuras del modelo integrado de gestión de la convivencia escolar. Los principales resultados indicaron una fuerte presencia de algunos elementos configuradores de la convivencia, como la comprensión por las demás personas y la interdependencia positiva, pero dicha presencia fue muy restringida en cuanto a los valores del pluralismo como elementos constituyentes de la convivencia. Si bien es cierto que algunas tipologías de conflictos escolares se encontraron en la formación inicial del profesorado, como los problemas de disciplina, el acoso entre iguales o la violencia, por el contrario, el tema del vandalismo, el acoso sexual o el plagio generalmente fueron inexistentes. En las titulaciones analizadas se encontraron diferentes estrategias, técnicas y herramientas para la gestión de la convivencia, pero no se encontraron incardinadas en un modelo integrador. Por todo ello, se puede concluir que la formación inicial del profesorado debe seguir mejorando en algunas direcciones concretas.

Palabras clave: convivencia escolar; formación docente; modelo integrado; planes de estudio; análisis documental.

\section{ABSTRACT}

School coexistence is a subject of interest that substantially conditions the types and quality of learning. But the management of school coexistence is not an easy question to address and requires, at least, an adequate teacher education. For this reason, the main aim of this research was to analyse what content, objectives, competencies and resources are being worked in the initial teacher education of Early Childhood and Primary Education. For this, the teaching guides (2019-2020) of the Spanish Faculties of Education positioned in the Shanghai Ranking (2224 guides studied) were analysed. The questions to be analysed revolved around: (1) the conceptualization and dimensionality of coexistence, (2) the configuration and typologies of school conflicts, and (3) the structures of the integrated model of management of school coexistence. The main results showed a strong presence of some shaping elements of coexistence, such as understanding by other people and positive interdependence, but the presence was very restricted in terms of values of pluralism as constituent elements of coexistence. Although it is true that some types of school conflicts were founded in the initial teacher education, such as discipline problems, bullying or violence, on the contrary, the subject of vandalism, sexual harassment or plagiarism were generally non-existent. In the analysed degrees, different strategies, techniques and tools for coexistence management were found, but they were not included in an integrative model. For all these reasons, it can be concluded that initial teacher education should continue to improve in some specific directions.

Key words: school coexistence; teacher education; integrated model; study plans; documental analysis. 
CARLOS MONGE-LÓPEZ Y PATRICIA GÓMEZ-HERNÁNDEZ

EL PAPEL DE LA CONVIVENCIA ESCOLAR EN LA FORMACIÓN INICIAL DEL PROFESORADO DE EDUCACIÓN INFANTIL Y PRIMARIA

\section{INTRODUCCIÓN}

La gestión de la convivencia escolar supone un asunto de especial importancia en los centros educativos en cuanto que, entre otras cuestiones, determina el tipo y calidad de los aprendizajes, las condiciones de vida dentro de la organización escolar, el desarrollo socio-afectivo o la adquisición de valores. De hecho, son varios los estudios e informes que alertan de la necesidad de seguir trabajando y mejorando el tema de la convivencia en los centros educativos, ya sean a nivel internacional (Gázquez, Pérez-Fuentes y Carrión, 2011; Gázquez, Pérez-Fuentes, Lucas y Fernández, 2011; Peiró, 2012), nacional (Díaz-Aguado, 2010; Zabalza, 2002; Zaitegi, 2010) o autonómico (Lázaro-Visa y Fernández-Fuertes, 2018; Luengo, 2019; Tirado y Conde, 2016).

La convivencia es una cuestión que se enseña y aprende, ya sea explícita o implícitamente. Por ello, los centros educativos se constituyen como escenarios privilegiados e idóneos para promover procesos de enseñanza-aprendizaje centrados en la idea de convivencia (Delors, 2013). Y esto, como recogen Anderson y Cohen (2015), implica que el profesorado es el agente que termina diseñando y desarrollando un currículo centrado en la convivencia, además de ser tradicionalmente visto como aquel que gestiona directamente el clima de aula y las relaciones con el alumnado y con algunos miembros de la comunidad educativa. De aquí se puede desprender la necesidad de formar al profesorado en la enseñanza de la convivencia.

A pesar de su importancia, la gestión de la convivencia en los centros educativos es un tema complejo de abordar debido a sus distintas interpretaciones, a las distintas formas de abordar la convivencia escolar, a los numerosos elementos que la constituyen, a los cuantiosos factores que influyen sobre la misma, etc. Como afirman García-Raga y López-Martín (2011), la construcción colectiva de la convivencia supone la principal dificultad para llegar a una conceptualización ampliamente aceptada. En este sentido, la formación docente supone un elemento fundamental para dotar al profesorado de conocimientos, capacidades y herramientas en aras de poder construir una convivencia favorable y prevenir e intervenir educativamente sobre los conflictos.

El objetivo principal de esta investigación es analizar qué contenidos, objetivos, competencias y recursos se están trabajando en la formación inicial del profesorado en torno a la convivencia escolar a partir del análisis de las guías docentes (2019-2020).

\section{CONVIVENCIA ESCOLAR, CONFLICTO Y HERRAMIENTAS PARA SU PREVENCIÓN Y RESOLUCIÓN}

Partiendo de una definición amplia y global de convivencia escolar estrechamente vinculada con el deseo de paz, la convivencia no puede entenderse simplemente como una respuesta de prevención a la violencia, ya que supone un 
proyecto ilusionante y un compromiso colectivo por querer vivir con y para los otros en torno a una meta o proyecto común, lo que implica generar procesos sociales de participación orientados a la creación de un sentido positivo de pertenencia a la institución, lo que lleva a caracterizar este concepto como "convivencia pacífica" conectada con el conocimiento útil sobre gestión de conflictos y recogiendo que el conflicto forma parte de la vida (Torrego, 2012). No obstante, no hay que olvidar los antecedentes histórico-conceptuales del constructo de convivencia, yendo desde su inclusión dentro del clima social escolar, donde se destaca el papel de las relaciones interpersonales, pasando por los estudios iniciales acerca del maltrato entre iguales, donde predominan las relaciones de dominio-sumisión, y avanzando hasta los cambios promovidos por las administraciones educativas a través de normativas específicas y esto lleva a una evolución del término y de su conceptualización (Córdoba, Del Rey y Ortega-Ruiz, 2014, p. 213):

La convivencia escolar se define como el entramado de relaciones interpersonales que surgen entre los miembros de la comunidad educativa, cuando éstos interactúan en la vida diaria a través de las actividades comunes, la comunicación que los relaciona y las directrices educativas que los organizan. Creencias, actitudes y valores, se articulan en un proceso complejo que es asumido y aceptado por todos los miembros de la comunidad como compartido.

Desde algunos resultados alcanzados en el contexto español, la convivencia escolar puede definirse como aquel término constituido principalmente por la gestión interpersonal positiva, la red social de iguales, la disciplina y el cuidado docente, los cuales se alejan de las agresiones, la victimización y, entre otros aspectos, la disrupción (Del Rey, Casas y Ortega-Ruiz, 2017). Y, por su parte, algunos resultados hallados con muestras internacionales vienen a conceptualizar la convivencia escolar como el conjunto de relaciones interpersonales y de participación educativa opuestos a la indisciplina, al acoso escolar, a las conductas antisociales, disruptivas y de desmotivación y, al menos, al desinterés académico (Gázquez, et al., 2011). Otra definición de convivencia extraída a partir de diversas propuestas sugieren que es aquella que «se construye día a día con el establecimiento de unas relaciones consigo mismo, con las demás personas y con el entorno [...] fundamentadas en la dignidad humana, en la paz positiva y en el respeto a los Derechos Humanos.» (Uruñuela, 2018, p. 35).

En el marco de los cuatro pilares de la educación formulados por la UNESCO (Delors, 2013), se habla de aprender a conocer, aprender a hacer, aprender a ser y aprender a convivir, considerando que este último pilar se compone de los siguientes elementos: el desarrollo de la comprensión de las demás personas, el despliegue de formas positivas de interdependencia (realización de proyectos comunes y preparación para la gestión de conflictos), el respeto de los valores del pluralismo y la búsqueda de la paz. En este contexto del aprendizaje de la convivencia, se viene evidenciando la relación entre la empatía y la capacidad para 

DE EDUCACIÓN INFANTIL Y PRIMARIA

la gestión de conflictos en el ámbito educativo (Hastings, Kavookjian y Ekong, 2019). Por su parte, el despliegue de formas positivas de interdependencia lleva a considerar el aprendizaje cooperativo como marco protector de la convivencia (Torrego, 2006), entender la convivencia como dimensión configuradora de la participación educativa (Torrego, et al., 2019) y, entre otras cuestiones, fomentar la construcción de una comunidad educativa comprometida con los valores democráticos (Grau, García-Raga y López-Martín, 2017). Cabe añadir el respecto a los valores del pluralismo dentro del aprendizaje de la convivencia, entendiendo la diversidad como un elemento positivo y favorecedor del aprendizaje de la convivencia, entendiendo la diversidad en una mirada amplia que incluya, al menos, diferencias sociales, culturales, religiosas, generacionales, sexuales, ideológicas, económicas, etc. (Arrase y Zarraute, 2019; Buckworth, 2019; Engebretson, en prensa; Teff-Seker, 2020). A pesar de estas cuestiones, quizá la idea más difundida sobre el aprendizaje de la convivencia sea aquella relacionada con la búsqueda de la paz (Galtung, 2003; Johnson, 2019; Teff-Seker, 2020; Torrego, 2012), especialmente en lo relativo a evitar la violencia (peacekeeping), resolver conflictos pacíficamente (peacemaking) y construir la paz (peacebuilding) (Bickmore, 2019). Desde un marco competencial, el aprendizaje de la convivencia implica (GarcíaRaga y López-Martín, 2011): saber participar, saber cooperar, ser responsables, ser tolerantes, disponer de habilidades sociales, ser solidarios y aprender a resolver pacíficamente los problemas interpersonales.

Pero hablar de convivencia lleva necesariamente a abordar también el tema de los conflictos escolares. En este sentido, un conflicto puede entenderse como aquella «situación en la que dos o más personas o grupos [(incluyendo instituciones, administraciones y escuelas)] perciben o tienen posiciones, valores, intereses, aspiraciones, necesidades o deseos contrapuestos.» (Uruñuela, 2018, p. 44). En líneas generales, la teoría sobre conflictología viene identificando tres componentes fundamentales de los conflictos (Galtung, 2003): conductas, actitudes y contradicciones. Las conductas hacen referencia al elemento objetivo y visible de los conflictos, siendo el comportamiento que puede ser observado. Por su parte, las actitudes son el elemento motivacional de los conflictos en torno a cómo piensan y sienten las partes implicadas. Y las contracciones son entendidas como el elemento subjetivo en relación con el tema del conflicto.

Otra cuestión por destacar para la comprensión de este tema es la tipología de conflictos de convivencia escolar, si bien es cierto que en ocasiones son denominados "dificultades para la convivencia escolar» (Gázquez, et al., 2011). A pesar de no ser la única propuesta, Torrego (2006) realiza una clasificación muy completa sobre los principales conflictos en el ámbito educativo:

- Violencia general. Es el conjunto de conductas violentas que incluyen violencia psicológica, física o estructural. La violencia psicológica general implica el maltrato entre iguales, las faltas de respeto, la intimidación y la 
exclusión social. La violencia física puede ser ejercida hacia uno mismo o hacia los demás. Y la violencia estructural hace referencia a las conductas violentas que pueden ser desencadenadas por las políticas educativas injustas, la propia estructura y funcionamiento inadecuado de la organización escolar y el diseño curricular poco democrático.

- Disrupción. Hace referencia al conjunto de conductas no apropiadas dentro del aula que ralentizan y/o impiden el desarrollo y funcionamiento esperado de la clase.

- Vandalismo. Puede entenderse como la violencia física que desemboca en daños al centro educativo y a sus instalaciones.

- Problemas de disciplina. Es la tipología de conflicto de convivencia que conlleva al incumplimiento de las normas, bien sean del centro o, simplemente, del aula.

- Acoso entre iguales o bullying. Es un tipo de maltrato que realiza un alumno o varios contra otro compañero. Para hablar de acoso entre iguales se deben cumplir los siguientes criterios: se ha de producir de manera horizontal, se debe prolongar a lo largo del tiempo, se produce cuando el alumno maltratado no tiene la capacidad adecuada para defenderse, conlleva un ataque físico y/o psicológico, debe existir intención de infligir daño y conlleva el deterioro de la integración social y de los aprendizajes.

- Acoso y abuso sexual. Puede considerarse un atentado sobre la libertad sexual y dignidad de las personas y una manifestación de comportamiento antisocial oculto. Se considera que son un conjunto de conductas no consentidas por ambas partes, o que implican desigualdad de poder, y que hacen referencia a tocamientos, relaciones sexuales forzadas, caricias inadecuadas, contacto sexual perverso, exposición indecente, masturbación exhibicionista, exposición directa a la pornografía, explotación sexual, vejaciones sexuales, etc.

- Desafección, absentismo y abandono. La desafección escolar hace referencia al comportamiento de ausencia emocional que conlleva a la pérdida de sentido y ruptura con la oferta educativa. El absentismo escolar se produce cuando el alumno falta a clase de forma reiterada, ya sea de forma justificada o injustificada. Y el abandono escolar consiste en la ausencia definitiva e injustificada al centro escolar sin haber terminado la etapa educativa que se está cursando.

- Fraude, corrupción, copia y plagio. Se trata de un conjunto de conductas inapropiadas socialmente dentro de la gestión y el desarrollo del desempeño educativo. 

DE EDUCACIÓN INFANTIL Y PRIMARIA

- Problemas de seguridad. Son el conjunto de conductas internas o externas a los miembros del centro educativo que ocasionan un clima de miedo a sufrir daños.

No obstante, cabe tener en cuenta que no todos los conflictos escolares tienen la misma importancia, prevalencia e incidencia (Díaz-Aguado, 2010; Luengo, 2019). Y, por lo tanto, las estrategias de prevención y resolución no pueden ser iguales (Gázquez, et al., 2011; Peiró, 2012).Si bien es cierto que existen distintos modelos de gestión de la convivencia de gran interés e impacto (Bickmore, 2019), aquí se recurre al modelo integrado de gestión de la convivencia escolar con estrategias, técnicas y herramientas con resultados contrastados, el cual se estructura en torno a tres pilares básicos (Torrego, 2006):

1. Elaboración democrática de normas. A partir de la elaboración democrática de normas lo que se pretende, fundamentalmente, es generar compromiso con la mejora de la convivencia a partir del análisis o toma de conciencia de la situación en la que se encuentra el centro, así como aportar propuestas y normas, velar por su regulación y, entre otras cuestiones, realizar seguimientos y evaluaciones de las actividades y normas propuestas. El proceso para la gestión democrática de normas puede sintetizarse de la siguiente forma (Uruñuela, 2018): fase de diseño (reflexión inicial, intercambio de ideas, toma de decisiones e información a la comunidad educativa), fase de sensibilización (actividades específicas para el alumnado, para el profesorado, para las familias y para el personal no docente), fase de creación de normas y protocolos de intervención ante su incumplimiento (creación con los alumnos; creación con el profesorado, las familias y el personal no docente; consenso y acuerdos) y fase de aplicación, institucionalización y seguimiento, que es lo que da pie a volver a empezar este ciclo. La elaboración democrática de normas correlaciona negativamente con la violencia escolar, la disrupción en el aula y la exclusión (Álvarez-García, Dobarro, Rodríguez, Núñez y Álvarez, 2013). Y cuando se trata de diseños cuasi-experimentales ofrece un adecuado tamaño del efecto para cuestiones relacionadas con la puntualidad, la atención y el ruido en clase (Carmona, Buendía y Gallego, 2016).

2. Equipo de tratamiento y resolución de conflictos, que integra los Programas de Mediación Escolar y de Alumnos Ayudantes. El primero de ellos consiste en ofrecer un sistema voluntario y confidencial para la gestión de conflictos con la ayuda de una tercera persona neutral (el mediador) donde se busca satisfacer las necesidades de las partes a partir de la regulación pautada del proceso de comunicación, mientras que el segundo programa consiste en la creación de redes de apoyo entre el alumnado, contando con alumnos seleccionados y formados para la detección y atención de problemas 

DE EDUCACIÓN INFANTIL Y PRIMARIA

aportando confianza y solidaridad (Torrego, 2006). Dada la trayectoria de estos programas, existen evidencias suficientes sobre su impacto que son analizadas en varios meta-análisis (Dymnick, Weissberg y Henry, 2011; Moeyaert, Klingbeil, Rodabaugh y Turan, en prensa; Turk, 2018).

3. Marco protector de la convivencia. Se refiere al conjunto de medidas organizativas y curriculares que vienen demostrando su impacto en el aprendizaje de la convivencia, siento los elementos más destacados el estilo democrático para el afrontamiento de la disrupción (Tirado y Conde, 2016), el aprendizaje cooperativo (Bilgin, Karakuyu, Tatar y Cetin, 2012; León, Polo, Gozalo y Mendo, 2016; Ryzin y Roseth, 2018), el aprendizaje-servicio (Ochoa y Pérez, 2019; Pérez y Ochoa, 2017; Torrego, et al., 2019), la acción tutorial centrada en la convivencia (Verdeja, 2012), las estrategias específicas centradas en la convivencia (Vega, 2017) y, entre otros ejemplos, el diseño, desarrollo y evaluación del Plan de Convivencia (Merma-Molina, Ávalos y Martínez, 2019; Tirado y Conde, 2016).

Sin embargo, el aprendizaje de la convivencia, su gestión y la implementación de herramientas efectivas para la prevención y resolución de conflictos suponen una cuestión con mucha complejidad que requiere, al menos, una adecuada formación del profesorado. De hecho, Phillips (2010) sostiene que la adecuada formación del profesorado debe llevar a la gestión de los conflictos internos y externos para lograr un correcto ajuste psicológico y un verdadero desarrollo personal y profesional.

\section{FORMACIÓN DEL PROFESORADO EN TORNO A LA CONVIVENCIA ESCOLAR}

La investigación sobre esta temática parece desigual en función de la tipología de conflicto de convivencia o de las medidas a adoptar. En líneas generales, se puede señalar que los docentes en activo y los estudiantes de Magisterio reconocen que la formación inicial del profesorado es insuficiente en cuanto a gestión de la diversidad y de los conflictos, e incluso, en algunos casos, la formación recibida es percibida como sexista, inadecuada y alejada de herramientas prácticas (Gómez-Jarabo, 2015). No obstante, a pesar de estas deficiencias en la formación del profesorado, Elbaz-Luwisch (2009) viene mostrando cómo el uso pedagógico de los conflictos en futuros maestros es una herramienta para la transformación y la mejora en escuelas multiculturales. En esta línea, otra cuestión a destacar puede ser que la gestión de los conflictos internos suele ser una de las cuestiones más olvidadas en la formación docente, especialmente, como se muestra a partir de dos casos, en la formación inicial del profesorado (Phillips, 2010).

Quizá el tema del bullying en la formación del profesorado sea uno de los conflictos más abordados en la investigación. Así, algunos resultados indican la 

DE EDUCACIÓN INFANTIL Y PRIMARIA

existencia de una estrecha relación entre la formación específica recibida y la autopercepción para identificar casos de acoso entre iguales e implementar protocolos de actuación (Goryl, Neilsen-Hewett y Sweller, 2013), así como con el número de casos de bullying en contextos específicos (Domeneci, 2017) o, entre otros aspectos, las competencias docentes para la caracterización de este fenómeno, la identificación de los agentes implicados y las estrategias eficaces de afrontamiento (Benítez, García-Berbén y Fernández-Cabezas, 2009). Sin embargo, a pesar de su importancia demostrada, el profesorado de diversos contextos señala que su formación en torno a este fenómeno es deficiente, lo que dificulta su conceptualización, identificación e intervención (Silva y Rosa, 2013).

De igual forma, la formación docente en materia de violencia escolar suele ser uno de los temas más estudiados, quizá incluso más que el bullying. Pero cabe señalar la gran diversidad de tipologías de violencia que se pueden producir en los centros educativos. Así, por ejemplo, se puede hablar de ciber-violencia (Nagle, 2018), violencia de género (Arrese y Zarruate, 2019), violencia sexual (Engebretson, 2019), violencia simbólica (Buckworth, 2019; Toshalis, 2010), violencia psicológica (Rodger, et al., en prensa) o violencia estructural (Sawa, et al., 2015) en la formación docente. A pesar de esta gran diversidad de conductas violentes y de los contextos específicos desde las que se abordan, la mayoría de los estudios señalados coinciden en la necesidad de seguir trabajando para mejorar la formación docente en esta materia, haciéndolo prioritariamente en los primeros estadios de formación.

Sin embargo, otros tipos de conflictos de convivencia en la formación del profesorado quedan mucho menos investigados. De hecho, una de las características del abuso sexual a menores es su escasa presencia en el currículo de los futuros docentes, dificultando así su prevención (Mathews, 2011; Scholes, Jones, StielerHunt, Rolfe y Pozzebon, 2012). Otra temática poco investigada hasta ahora hace referencia a la formación del profesorado en materia de abandono escolar, en cuanto que los datos indican la presencia de factores predictivos del abandono incluso en los estudiantes de titulaciones conducentes a la función docente (Bohndick, 2020). Incluso, mucha menos atención suele recibir la investigación sobre formación del profesorado en materia de prevención e intervención sobre la corrupción, a pesar de ser uno de los grandes males de varios países (Reyes, 2007), o sobre la falta de disciplina en las aulas, siendo una de las preocupaciones más reiteradas del profesorado (Merrett y Wheldall, 2017).

Como se viene recogiendo, si bien es cierto que la investigación sobre formación docente en materia de convivencia suele centrarse en la conceptualización e identificación de determinados conflictos, los estudios de impacto sobre la prevención y resolución constructiva de los mismos, así como sobre el aprendizaje de la paz y la interdependencia, pueden resultar escasos. Retomando algunas de las 

DE EDUCACIÓN INFANTIL Y PRIMARIA

estructuras del modelo integrado de gestión de la convivencia escolar (Torrego, 2006), la creación de un aula gestionada democráticamente ayuda a crear un clima de convivencia y contribuye a un verdadero cambio actitudinal, filosófico y pedagógico del profesorado, pero algunos docentes se ven obligados a auto-formarse para poder hacerlo porque la oferta formativa oficial resulta insuficiente (Brubaker, 2012). Dentro del marco protector de la convivencia, los estilos de afrontamiento democrático de la disrupción suelen estar más presentes en profesores noveles que en profesores experimentados (Peñalva, Hernández y Guerrero, 2013), por lo que es importante incorporar esta cuestión en el marco competencial de la formación inicial del profesorado. Y, por lo que se refiere a los equipos de tratamiento y resolución de conflictos, se puede evidenciar que los futuros docentes admiten que su formación en torno a los programas de mediación escolar y programas de alumnos ayudantes es escasa (Álvarez-García, Rodríguez, González-Castro, Núñez y Álvarez, 2010).

Un estudio realizado en el contexto español de la formación inicial del profesorado en materia de gestión de conflictos pone de manifiesto algunos datos interesantes (López, Tejero y Fernández, 2017). Concretamente, se puede destacar que el $53.5 \%$ de los estudiantes de titulaciones conducentes a la función docente afirma no tener materias optativas sobre tratamiento de conflictos escolares. El $45.5 \%$ dice no abordar el abordar el componente emocional de los conflictos durante su formación. El 67.4 \% indica no haber recibido información sobre la legislación en materia de conflictos escolares. El $47.6 \%$ afirma desconocer los procesos de mediación de conflictos, mientras que solamente el $8.9 \%$ reconoce haber participado con frecuencia en ellos. El 56.5 \% indica que desde la formación inicial del profesorado no se impulsa la reflexión sobre los aspectos ocultos de los conflictos, y el $54.5 \%$ señala no haber analizado conflictos derivados del desarrollo curricular. Finalmente, entre otros hallazgos, se puede destacar que el $68.3 \%$ manifiesta que la presencia de materias sobre convivencia escolar en su formación inicial es inadecuada.

Siendo conscientes de la existencia de algunas experiencias sobre formación inicial del profesorado en materia de gestión de la convivencia escolar (García-Bacete, 2009; Johnson, 2019; Negro, et al., 2017), se trata de situaciones concretas que no se dan de forma sistemática en la globalidad y amplitud del contexto español. De hecho, tras el análisis de los planes de estudios de la formación inicial del profesorado en todas las universidades españolas tras la implementación del Plan Bolonia, no se llegan a identificar más de cinco asignaturas específicas sobre la construcción de la convivencia escolar (Manso, 2019).

A pesar de todas estas evidencias, la investigación en materia de formación docente en torno a la convivencia escolar suele hacerse desde la perspectiva de sus protagonistas, en cuanto que no se suelen encontrar investigaciones de impacto que analicen las políticas educativas ni el diseño curricular en la formación inicial del profesorado sobre este tema. 


\section{MÉTODO}

Esta investigación se desarrolló desde un enfoque cualitativo con análisis documental de las guías docentes de los Grados en Educación Infantil y Educación Primaria. Para ello se seleccionaron las universidades españolas, tanto públicas como privadas, incluidas en el Academic Ranking of Word Universities (conocido como Shanghai Ranking) en el área de Educación de 2019 (Tabla 1).

TABLA 1

Universidades españolas en el Shanghai Ranking de Educación (2019)

\begin{tabular}{|l|l|l|l|l|l|l|}
\hline & $\begin{array}{l}\text { Posición } \\
\text { mundial }\end{array}$ & $\begin{array}{l}\text { Publicaciones } \\
\text { indexadas }\end{array}$ & $\begin{array}{l}\text { Citas } \\
\text { recibidas }\end{array}$ & $\begin{array}{l}\text { Colaboración } \\
\text { internacional }\end{array}$ & $\begin{array}{l}\text { Mejores } \\
\text { artículos }\end{array}$ & Premios \\
\hline UB & $151-200$ & 49.6 & 65.3 & 58.8 & 23.6 & - \\
\hline EHU/UPV & $201-300$ & 43.6 & 72.2 & 36.5 & 13.6 & - \\
\hline UAM & $201-300$ & 34.6 & 69.2 & 67.7 & 19.2 & - \\
\hline US & $201-300$ & 41.1 & 65.8 & 43.6 & 13.6 & - \\
\hline UAB & $301-400$ & 45.5 & 63.3 & 59.1 & 0.0 & - \\
\hline UGR & $301-400$ & 46.3 & 62.2 & 55.2 & 0.0 & - \\
\hline UNED & $301-400$ & 36.2 & 73.6 & 44.8 & 0.0 & - \\
\hline USAL & $301-400$ & 33.5 & 64.7 & 64.7 & 13.6 & - \\
\hline UV & $301-400$ & 44.7 & 62.1 & 44.2 & 0.0 & - \\
\hline UAH & $401-500$ & 24.8 & 90.1 & 54.7 & 0.0 & - \\
\hline UCLM & $401-500$ & 24.8 & 67.5 & 47.3 & 19.2 & - \\
\hline UCM & $401-500$ & 38.2 & 50.1 & 53.3 & 0.0 & - \\
\hline UC3M & $401-500$ & 23.6 & 94.9 & 68.8 & 0.0 & - \\
\hline UDG & $401-500$ & 21.7 & 67.4 & 51.7 & 19.2 & - \\
\hline ULL & $401-500$ & 25.4 & 70.7 & 55.1 & 13.6 & - \\
\hline UMA & $401-500$ & 30.4 & 57.7 & 41.6 & 13.6 & - \\
\hline UNICAN & $401-500$ & 24.2 & 62.2 & 59.4 & 13.6 & - \\
\hline UNIOVI & $401-500$ & 33.0 & 77.1 & 63.3 & 0.0 & - \\
\hline UNIZAR & $401-500$ & 28.3 & 80.8 & 59.8 & 0.0 & - \\
\hline UOC & $401-500$ & 28.5 & 71.1 & 60.6 & 0.0 & - \\
\hline UPV & $401-500$ & 30.1 & 76.1 & 52.8 & 0.0 & - \\
\hline UVA & $401-500$ & 27.0 & 77.5 & 57.5 & 0.0 & - \\
\hline & & & & & & - \\
\hline
\end{tabular}

Fuente: Elaboración propia

(Nota: las universidades en el mismo rango de la clasificación han sido ordenadas alfabéticamente) 
Una vez seleccionadas las universidades se procedió a la búsqueda y compilación de todas las guías docentes de los Grados en Educación Infantil y Educación Primaria del curso 2019-2020 (Tabla 2), considerando un total de 2.224 documentos analizados.

TABLA 2

Guías docentes utilizadas en el análisis documental

\begin{tabular}{|c|c|c|c|}
\hline & Ed. Infantil & Ed. Primaria & Total \\
\hline UB & 69 & 88 & 157 \\
\hline EHU/UPV & 57 & 57 & 114 \\
\hline UAM & 64 & 82 & 146 \\
\hline US & 42 & 51 & 93 \\
\hline UAB & 56 & 78 & 134 \\
\hline UGR & 48 & 53 & 101 \\
\hline UNED & 0 & 0 & 0 \\
\hline USAL & 60 & 72 & 132 \\
\hline UV & 38 & 76 & 114 \\
\hline UAH & 53 & 64 & 117 \\
\hline UCLM & 56 & 65 & 121 \\
\hline $\mathrm{UCM}$ & 54 & 73 & 127 \\
\hline $\mathrm{UC} 3 \mathrm{M}$ & 0 & 0 & 0 \\
\hline UDG & 82 & 81 & 163 \\
\hline ULL & 48 & 55 & 103 \\
\hline UMA & 40 & 63 & 103 \\
\hline UNICAN & 62 & 69 & 131 \\
\hline UNIOVI & 49 & 77 & 126 \\
\hline UNIZAR & 46 & 75 & 121 \\
\hline UOC & 0 & 0 & 0 \\
\hline UPV & 0 & 0 & 0 \\
\hline UVA & 46 & 75 & 121 \\
\hline Total & 970 & 1254 & 2224 \\
\hline
\end{tabular}

Fuente: Elaboración propia

(Nota: existen algunos casos donde no de analizan las guías docentes porque en 2019-2020 no se ofertan las titulaciones que son objeto de esta investigación) 
CARLOS MONGE-LÓPEZ Y PATRICIA GÓMEZ-HERNÁNDEZ

EL PAPEL DE LA CONVIVENCIA ESCOLAR EN LA FORMACIÓN INICIAL DEL PROFESORADO DE EDUCACIÓN INFANTIL Y PRIMARIA

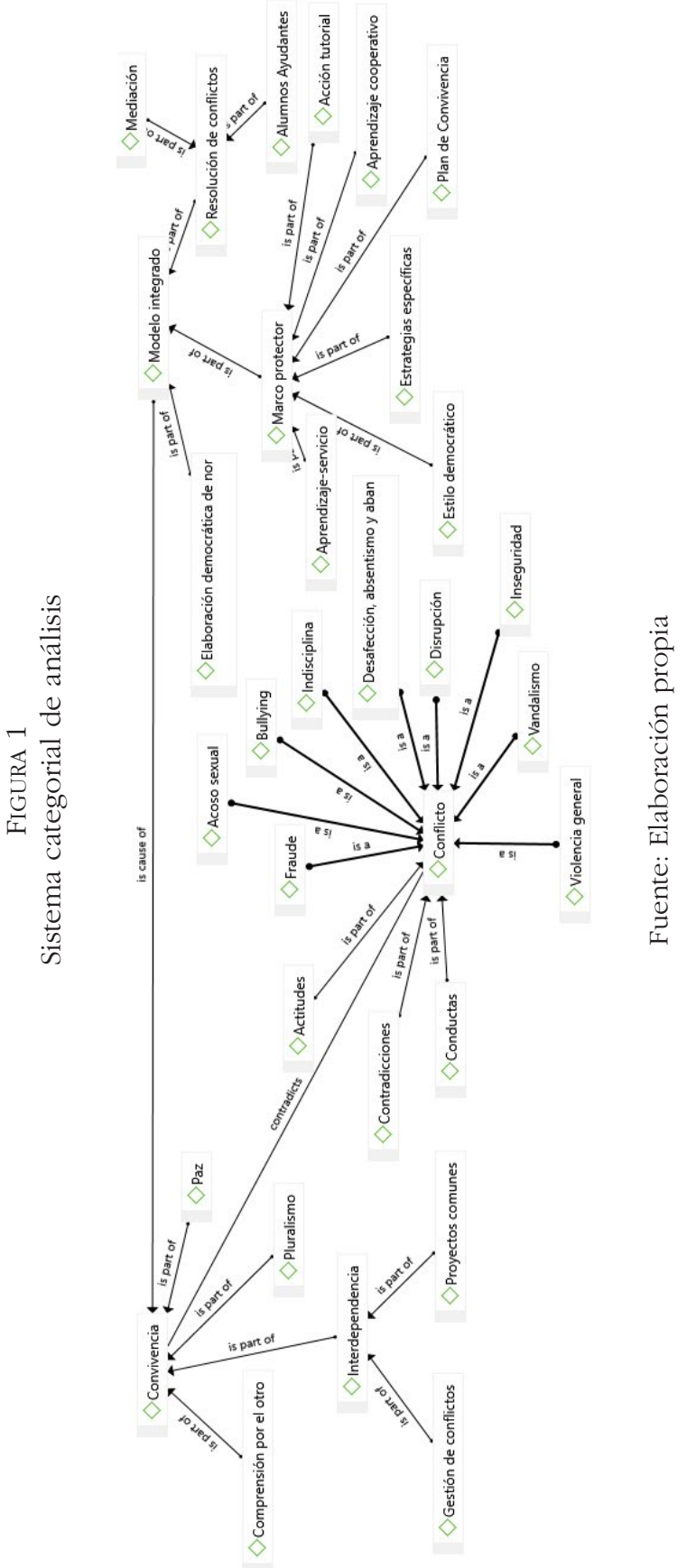



DE EDUCACIÓN INFANTIL Y PRIMARIA

Las guías docentes fueron estudiadas a través del análisis de contenido, para lo cual se empleó el software específico Atlas.ti 8. En dicho análisis se siguió un proceso de codificación y categorización centrado en las dimensiones configuradoras de la convivencia (Delors, 2013), las principales tipologías de conflictos de convivencia (Torrego, 2006), los componentes de los conflictos (Galtung, 2003) y las estructuras del modelo integrado de gestión de la convivencia (Torrego, 2006) (Figura 1). Se tuvo también en cuenta la tipología de asignaturas, los créditos de las mismas y los elementos curriculares referentes al citado sistema de códigos y categorías. Las guías docentes fueron analizadas en dos fases, contando con la participación de dos investigadores. En la primera fase, a través del buscador de palabras de Atlas.ti, se procedió a buscar términos claves de cada dimensión (p.ej.: mediación, mediado o mediar) para determinar si los fragmentos encontrados suponían una evidencia a incluir en el análisis, lo cual fue realizado por los investigadores por separado. Solamente en tres casos no se llegó a un consenso y se consultó a un experto reconocido en la materia. Posteriormente, en la segunda fase, los investigadores fueron leyendo conjuntamente cada documento para detectar y discutir la pertinencia de incorporar nuevas evidencias en base al sistema categorial establecido. Finalmente, se pasó a ordenar por frecuencia o aparición las categorías y subcategorías de cara a redactar este informe de resultados.

\section{Resultados}

\subsection{Dimensiones configuradoras de la convivencia}

Principalmente, la comprensión por las demás personas se reflejó en torno a la empatía. Esta cuestión se vino recogiendo en diversas asignaturas, tanto optativas como básicas y obligatorias, de ambas titulaciones. Generalmente, además de situarse en la contextualización de las asignaturas, la empatía resultó ser un término bastante recurrente que se pudo encontrar especialmente en las competencias (p. ej.: «mostrar empatía, sociabilidad y tacto en la comunicación con los demás» de la asignatura Acción tutorial del Grado en Infantil de la Universidad de Barcelona) y resultados de aprendizaje (p. ej.: «saber empatizar con las diferentes opciones ideológicas y vitales que integran las comunidades" de la asignatura Estrategias de mediación del Grado en Educación Primaria de la Universidad Autónoma de Barcelona) de la mayoría de las universidades analizadas.

Si bien es cierto que la interdependencia apareció en algunas asignaturas básicas (p.ej.: el objetivo de aprendizaje dirigido a «adquirir las nociones de interdependencia y sostenibilidad, así como los valores de solidaridad, justicia y tolerancia» de la asignatura Educación para la pazy la igualdad del Grado en Educación Primaria de la Universidad de Valladolid), su principal aparición se constató en la metodología de las asignaturas optativas del Grado en Educación Primaria que se vinculaban a las didácticas específicas ("la metodología cooperativa se estructura para asegurar 
interdependencia positiva, la interacción cara a cara, la responsabilidad individual y las habilidades inherentes a pequeños grupos» de la asignatura Innovación e investigación curricular en didáctica de la lengua y la literatura y de las Matemáticas del Grado en Educación Primaria de la Universidad de La Laguna). Por su parte, la gestión de conflictos como parte de la interdependencia se pudo observar con cierta diversidad de estrategias, herramientas y técnicas, pero esta dimensión se verá en apartados posteriores. Y la construcción de proyectos comunes como parte de la interdependencia supuso una cuestión presente casi exclusivamente en las asignaturas optativas de la Universidad de Cantabria en el marco de las competencias específicas (p.ej.: "valorar la importancia del trabajo en equipo, fomentando el aprendizaje cooperativo como herramienta para el desarrollo de la creatividad a través de proyectos comunes» de la asignatura Proyecto dramático y musical del Grado en Educación Infantil de la Universidad de Cantabria).

A pesar de que algunas asignaturas consideraron el pluralismo como dimensión configuradora de la convivencia (p.ej.: el contenido actitudinal vinculado a la «aceptación, valoración y reconocimiento de la diversidad y el pluralismo» de la asignatura Psicología social de la educación del Grado en Educación Infantil de la Universidad de Salamanca), esta idea no estuvo especialmente presente en asignaturas más allá del hecho religioso (p.ej.: el tema de contenidos acerca del "pluralismo religioso y multiculturalidad" de la asignatura Esencia del cristianismo del Grado en Educación Infantil de la Universidad Autónoma de Madrid).

De forma similar sucedió con las cuestiones referentes a la paz, estando fuertemente presentes en asignaturas vinculadas a la religión (p.ej.: la competencia dirigida a "promover los valores propios de una cultura de paz y de valores democráticos" de la asignatura Religión, cultura y valores del Grado en Educación Infantil de la Universidad de Castilla-La Mancha). No obstante, aquí sí que se ha encontrado una mayor presencia de la idea de paz como parte fundamental de la convivencia en algunas asignaturas básicas (p.ej.: la asignatura Educación para la paz y la igualdad del Grado en Educación Infantil de la Universidad de Valladolid), obligatorias (p. ej.: la competencia general destinada a «promover la educación democrática para la ciudadanía activa y una cultura de paz» de la asignatura Didáctica de las ciencias sociales del Grado en Educación Primaria de la Universidad de Granada) y optativas (p. ej.: el tema "educación para la paz» de la asignatura Diagnóstico y orientación psicopedagógica del Grado en Educación Primaria de la Universidad Autónoma de Madrid).

A pesar de esta presencia dimensional de la convivencia en la formación inicial del profesorado, no se encontró ninguna asignatura específica en cuyo título apareciese el término convivencia.

\subsection{Conflictos de convivencia escolar}

El desarrollo de actitudes estuvo muy presente en el análisis de los planes de estudio (p.ej.: actitudes hacia la enseñanza, actitudes hacia la atención a la diversidad o actitudes hacia determinas materias), pero esencialmente no se observó su 
CARLOS MONGE-LÓPEZ Y PATRICIA GÓMEZ-HERNÁNDEZ EL PAPEL DE LA CONVIVENCIA ESCOLAR EN LA FORMACIÓN INICIAL DEL PROFESORADO DE EDUCACIÓN INFANTIL Y PRIMARIA

relación explícita con su contribución en los conflictos escolares. De igual forma, la formación inicial del profesorado recogía cuestiones sobre valores, intereses y necesidades, aunque no solían estar directamente relacionados con los conflictos de convivencia.

La violencia general fue un tema bastante frecuente en las guías docentes de las titulaciones analizadas, aunque su frecuencia se redujo a asignaturas de carácter optativo. Aunque generalmente no se explicitó el tipo de violencia general (psicológica, física y estructural), cuando sí se hacía se solía hacer mayor referencia a la violencia psicológica. Concretamente, esta tipología se concentraba especialmente en las prácticas externas obligatorias a través de referencias al respeto por los demás (p. ej.: la competencia general referida a «reflexionar en grupo sobre la aceptación de normas y el respeto a los demás" del Prácticum en mención en lengua inglesa del Grado en Educación Infantil de la Universidad Autónoma de Madrid), así como en asignaturas troncales del área de Sociología de la Educación (p.ej.: el bloque de contenidos denominado "Estratificación, desigualdad y exclusión social» de la asignatura Procesos sociales básicos en educación del Grado en Educación Infantil de la Universidad de Sevilla).

La disrupción, el vandalismo, el acoso sexual, el abuso sexual y los problemas de seguridad fueron temáticas prácticamente inexistentes en la formación inicial del profesorado, donde, salvo casos muy aislados, no se encontraron evidencias en las guías didácticas más allá de algunas referencias bibliográficas específicas sobre estas tipologías de conflicto.

Aunque no estuvo presente de forma sustancial en todos los elementos curriculares, los problemas de disciplina sí se encontraron de forma frecuente en las competencias específicas de asignaturas de formación básica (p. ej.: «abordar y resolver problemas de disciplina» de la asignatura Sociología y psicología social de la educación del Grado en Educación Primaria de la Universidad de Zaragoza). Además, también pudo encontrarse en competencias específicas de asignaturas de obligatorias (p. ej.: "fomentar la convivencia fuera del aula y dentro de ella, resolver problemas de disciplina y contribuir a la resolución pacífica de conflictos» de la asignatura Didáctica del medio natural II del Grado en Educación Primaria de la Universidad de Cantabria) y de asignaturas optativas. En este caso, dentro de la oferta académica y opciones de optativas de las diferentes universidades, se pudieron destacar algunas asignaturas optativas específicas sobre esta materia (p. ej.: Intervención psicológica en problemas de disciplina del Grado en Educación Primaria de la Universidad de Salamanca) o asignaturas directamente relacionadas (p. ej.: Manejo del comportamiento problemático en el aula del Grado en Educación Primaria de la Universidad de Oviedo).

Entre todos los tipos de conflictos de convivencia, el bullying fue el más presente en las guías docentes analizadas de la formación inicial del profesorado, con diversidad de elementos curriculares y tipología de asignaturas, destacando aquellas de carácter optativo. 

DE EDUCACIÓN INFANTIL Y PRIMARIA

El tema del absentismo escolar sí resultó estar cubierto mediante los contenidos de asignaturas de formación básica, tanto en Educación Infantil (p. ej.: «alumnado en riesgo de marginación escolar y social: absentismo, acoso escolar y situación socioeconómica desfavorecida" de la asignatura Fundamentos psicológicos de atención a la diversidad de la Universidad de Alcalá) como en Educación Primaria (p. ej.: «absentismo, abandono y fracaso escolar en Europa, España y Andalucía: apuntes para su conocimiento y dimensión del fenómeno» de la asignatura Sociología de la educación de la Universidad de Granada). En cambio, el abandono escolar (salvo en dos ocasiones) y la desafección no se recogieron explícitamente en las guías docentes de las titulaciones analizadas.

Finalmente, las cuestiones sobre fraude, corrupción, copia y plagio se abordaron como meras advertencias u observaciones (p. ej.: «en la evaluación de tareas y examen se tendrán en cuenta la ortografía y la expresión, las normas de estilo de la APA y el plagio. [...] El fraude conlleva la calificación de suspenso» de la asignatura Aprendizaje, desarrollo y atención a la diversidad del Grado en Educación Primaria de la Universidad de Cantabria).

\subsection{Propuestas educativas para la gestión de la convivencia}

Si bien es cierto que a lo largo de las titulaciones analizadas se observaron algunas herramientas, técnicas y estrategias para la gestión de la convivencia, no se realizó desde un enfoque integrador que aglutinara esas respuestas en un mismo modelo de gestión de la convivencia escolar.

De igual forma, la elaboración democrática de normas resultó prácticamente inexistente en la formación inicial del profesorado. Se encontraron algunas evidencias sobre la reflexión grupal en torno a las normas de convivencia, pero se realizó únicamente desde la importancia del respeto a las mismas y no desde su construcción participativa (p. ej.: la competencia general referida a «reflexionar en grupo sobre la aceptación de normas y el respeto a los demás" de la asignatura optativa El entorno como recurso educativo del Grado en Educación Infantil de la Universidad Autónoma de Madrid).

A pesar de que la mediación de conflicto resultó ser la estrategia más frecuente, básicamente esta se dio en asignaturas optativas (p. ej.: la competencia dirigida a «identificar y diagnosticar los factores habituales de crisis familiar y social y desarrollar una capacidad de mediación para tratar con comunidades socioeducativas y resolver conflictos» de la asignatura Psicología de la educación familiar del Grado en Educación Infantil de la Universidad de Valladolid). En este caso, pudo destacarse la iniciativa de la Universidad Autónoma de Barcelona al ofertar una asignatura optativa específica sobre esta temática (Estrategias de mediación). En cambio, los programas de alumnos ayudantes como parte fundamental de los equipos de tratamiento de conflictos estuvieron mucho menos presentes en la formación inicial del profesorado, aunque, a diferencia de la mediación de conflictos, sí se encontraron algunos contenidos de aprendizaje en asignaturas de formación básica (p. ej.: «estrategias 
CARLOS MONGE-LÓPEZ Y PATRICIA GÓMEZ-HERNÁNDEZ EL PAPEL DE LA CONVIVENCIA ESCOLAR EN LA FORMACIÓN INICIAL DEL PROFESORADO DE EDUCACIÓN INFANTIL Y PRIMARIA

psicoeducativas para la promoción de la cooperación y la ayuda entre iguales» de la asignatura Educación para la paz y la igualdad del Grado en Educación Primaria de la Universidad de Valladolid).

En cuanto al marco protector de la convivencia escolar, pudo destacarse que la acción tutorial centrada en la convivencia y el diseño, desarrollo y evaluación del Plan de Convivencia estuvieron ampliamente presentes en las guías docentes analizadas, especialmente en aquellas referidas a la organización escolar y a las prácticas en centros educativos. A continuación, las siguientes estrategias más frecuentes resultaron ser el aprendizaje cooperativo y el aprendizaje-servicio, constatándose su presencia en asignaturas obligatorias, optativas y de formación básica, tanto a nivel metodológico (p. ej.: «la práctica consistirá en la intervención en centros mediante metodologías de aprendizaje-servicio» de la asignatura Didáctica de las ciencias experimentales II del Grado en Educación Primaria de la Universidad de Granada) como a nivel conceptual (p. ej.: la unidad temática sobre «aprendizaje colaborativo y cooperativo y corrección por pares» de la asignatura Didáctica de la lengua inglesa II del Grado en Educación Primaria de la Universidad Autónoma de Madrid). En el caso del aprendizaje cooperativo pudo reseñarse la existencia de asignaturas optativas específicas sobre esta cuestión (p. ej.: Aprendizaje cooperativo como estrategia de inclusión del Grado en Educación Primaria de la Universidad de Alcalá).

\section{DisCuSIÓN Y CONCLUSIONES}

Aunque son pocas las asignaturas específicas sobre construcción de la convivencia escolar en la formación inicial del profesorado a lo largo de todo el territorio español (Manso, 2019), en esta investigación no se encuentra ninguna. Esto puede deberse al carácter optativo de las mismas, lo que lleva a que algunas universidades ya no las oferten en el curso 2019-2020, como sucede en la Universidad de Cantabria, o a que directamente nunca han estado en su oferta formativa. La interdependencia positiva, la comprensión por lo demás, la búsqueda de la paz y los valores del pluralismo, como dimensiones configuradoras de la convivencia (Delors, 2013), se encuentran integradas a lo largo de los elementos curriculares de diversas asignaturas, lo cual lleva a un doble análisis. Por un lado, esto quiere decir que la convivencia escolar en la formación inicial del profesorado se está trabajando desde una perspectiva multidisciplinar, pero, en cambio, se puede correr el riego de diluir esta cuestión y que finalmente no sea abordada desde ninguna asignatura. No se puede olvidar la importancia de estas cuestiones en cuanto que la interdependencia positiva es uno de los elementos mejor valorados por los docentes en formación para la mejora de la convivencia y la inclusión (Negro, et al., 2017), así como que los estudiantes de titulaciones conducentes al desempeño de la docencia perciben la necesidad de utilizar la pluralidad como elemento de transformación y mejora escolar (Elbaz-Luwisch, 2009). En cualquier caso, dentro de esos elementos curriculares indicados, los resultados no terminan de ajustarse a propuestas competenciales sólidas (García-Raga y López-Martín, 2011). 
Los resultados hallados en esta investigación vienen a mostrar la escasa presencia de lo que Galtung (2003) denomina elementos invisibles del conflicto (actitudes y contradicciones) en los Grados de Educación Infantil y Primaria. Precisamente, esto supone un hecho anteriormente puesto de manifiesto desde el punto de vista del alumnado de estas titulaciones (López, et al., 2017).

Dado que los distintos tipos de conflictos de convivencia difieren entre sí en cuanto a manifestación, conceptualización, impacto o frecuencia (Díaz-Aguado, 2010; Luengo, 2019), en la formación inicial del profesorado no todos deben ser abordados con la misma importancia. En este sentido, puede observarse cómo el bullying es la tipología más frecuente en los planes de estudio analizados, pero, a pesar de ello, se considera deficiente (Silva y Rosa, 2013).

De igual forma, la violencia general también se encuentra presente en la formación inicial del profesorado, aunque en las guías docentes analizadas se puede reducir a asignaturas de carácter optativo en las que no todo el alumnado se matricula. Cuando se explicita el tipo de violencia general, sí existe una fuerte presencia de la violencia psicológica en elementos fundamentales de los planes de estudios, como es el Prácticum, aunque en algunos casos todavía se percibe como un elemento a mejorar (Rodger, et al., en prensa). Pero otros tipos de violencia, tal como vienen recogiendo Sawa et al. (2015) con la violencia estructural, se encuentran prácticamente olvidados.

Frente a temáticas como los problemas de disciplina o el absentismo escolar ya presentes en asignaturas básicas, específicas y optativas, otras tipologías de conflictos son prácticamente inexistentes. En este caso, puede destacarse la disrupción, el vandalismo, el acoso sexual, el abuso sexual, los problemas de seguridad, el abandono escolar, la desafección académica o, entre otras tipologías, el fraude, como se recoge en otros estudios previos en el caso del abandono (Bohndick, 2020) y de la corrupción (Reyes, 2007). Tras este análisis se puede llegar a dos claras conclusiones. La primera de ellas es que, como apuntan otros estudios (Elbaz-Luwisch, 2009; Gómez-Jarabo, 2015; Negro, et al., 2017; Phillips, 2010), la formación inicial del profesorado en materia de conflictos escolares es deficiente, pero es algo que no debe generalizarse a todos los tipos de conflictos. Y, en segundo lugar, dadas las dramáticas consecuencias generadas por el abuso sexual a menores (Mathews, 2011; Scholes, et al., 2012), cabe priorizar tipologías de conflicto como esta.

En líneas generales, a pesar de encontrar diversas herramientas para la gestión de la convivencia escolar durante la formación inicial del profesorado, se presentan de forma aislada y no se incardinan en propuestas globales e integradoras con resultados contratados como propone Torrego (2006). Dentro de esa propuesta, los resultados de esta investigación confirman lo señalado previamente por Brubaker (2012) al recoger la escasa presencia de la creación democrática de normas en estos contextos. Por su parte, aunque la mediación de conflictos resulta ser una de las estrategias más presentes en las titulaciones analizadas, se contextualiza fundamentalmente en asignaturas optativas, lo que ayuda a explicar algunos datos previamente expuestos (Álvarez-García, et al., 2010; López, et al., 2017). Y, si bien 
es cierto que el aprendizaje cooperativo puede ser recurrente en la formación inicial del profesorado, este recurso a veces es considerado como deficiente en materia de convivencia escolar (Negro, et al., 2017).

No obstante, todas estas conclusiones han de considerarse con precaución en cuanto a las debilidades metodológicas presentes en esta investigación. Concretamente, las guías didácticas analizadas corresponden a las Facultades de Educación españolas mejor posicionadas a nivel internacional, por lo que cabe considerar este sesgo para su generalización. A pesar de esto, si las mejores titulaciones de Educación Infantil y Primaria presentan ciertas necesidades para mejorar la formación inicial del profesorado en materia de convivencia escolar, quizá se pueda construir una pequeña hipótesis sobre lo que está sucediendo en las instituciones peor valoradas internacionalmente. Considerando estas debilidades metodológicas, las futuras líneas de investigación pueden ir encaminadas al análisis de guías didácticas de universidades que sí ofertan asignaturas específicas sobre convivencia en la formación inicial del profesorado de Educación Infantil y Primaria, además de incluir otras fuentes de información y técnicas de recogida de análisis para contrastar lo que se planifica con lo que realmente se desarrolla en la práctica.

\section{REFERENCIAS BIBLIOGRÁFICAS}

Álvarez-García, D., Dobarro, A., Rodríguez, C., Núñez, J. C. y Álvarez, L. (2013) El consenso de normas de aula y su relación con bajos niveles de violencia escolar. Infancia y Aprendizaje, 36(2), 199-217. https://doi.org/10.1174/021037013806196229

Álvarez-García, D., Rodríguez, C., González-Castro, P., Núñez, J. C. y Álvarez, L. (2010). Training of pre-service teachers in school coexistence and classroom management resources. European Journal of Education and Psychology, 3(2), 187-198. https://doi. org/10.30552/ejep.v3i2.56

Anderson, G. \& Cohen, M. I. (2015). Redesigning the identities of teachers and leaders: A framework for studying new professionalism and educator resistance. Education Policy Analysis Archives, 23(85), 1-25. https://doi.org/10.14507/epaa.v23.2086

Arrese, E. M. y Zarraute, V. F. (2019). La violencia de género en redes sociales como partida para la reflexión acerca de la coeducación en la formación inicial del profesorado. Pensamiento Educativo. Revista de Investigación Educacional Latinoamericana, 56(1), 1-16. https://doi.org/10.7764/PEL.56.1.2019.7

Benítez, J. L., García-Berbén, A. y Fernández-Cabezas, M. (2009). The impact of a course on bullying within the pre-service teacher training curriculum. Electronic Journal of Research in Educational Psychology, 7(1), 191-208. https://doi.org/10.25115/ejrep.v7i17.1318

Bickmore, K. (2019). Conflict resolution education: Multiple options for contributing to just and democratic peace. En W. J. Pammer \& J. Killian (Eds.), Handbook of conflict management (pp. 3-32) (2. ${ }^{a}$ ed.). Marcel Dekker.

Bilgin, I., Karakuyu, Y., Tatar, E. \& Cetin, A. (2012). The effects of cooperative learning techniques on 5th grade students' conflict resolution and empathic tendencies in Science and Technology Course. New Educational Review, 27(1), 184-194. 

DE EDUCACIÓN INFANTIL Y PRIMARIA

Bohndick, C. (2020). Predictors of dropout intentions in teacher education programmes compared with other study programmes. Journal of Education for Teaching, 46(2), 207-219. https://doi.org/10.1080/02607476.2020.1724652

Brubaker, N. D. (2012). Multiple layers of self in an evolving pedagogy of teacher education: conflict and compromise in a quest for classroom democracy. Studying Teacher Education, 8(1), 3-18. https://doi.org/10.1080/17425964.2012.657009

Buckworth, J. (2019). Symbolic violence in teacher education: embracing cultural diversity or cultural discrimination? Asia-Pacific Journal of Teacher Education, 47(3), 309-321. https://doi.org/10.1080/1359866X.2018.1548691

Carmona, M., Buendía, L. y Gallego, A. (2016). La elaboración democrática de las normas de convivencia en el aula: una estrategia participativa. International Journal of Developmental and Educational Psychology, 1(1), 341-354. https://doi.org/10.17060/ijodaep.2016. n1.v1.171

Córdoba, F., Del Rey, R. y Ortega-Ruiz, R. (2014). Convivencia escolar en España: una revisión histórico-conceptual. Revista Confluencias. Análisis, Experiencias y Gestión de Conflictos, 2(2), 199-221.

Del Rey, R., Casas, J. A. y Ortega-Ruiz, R. (2017). Desarrollo y validación de la Escala de Convivencia Escolar (ECE). Universitas Psychologica, 16(1), 1-11. https://doi.org/10.11144/ Javeriana.upsy16-1.dvec

Delors, J. (2013). The treasure within: learning to know, learning to do, learning to live together and learning to be. What is the value of that treasure 15 years after its publication? International Review of Education, 59(3), 319-330. https://doi.org/10.1007/s11159-013-9350-8

Díaz-Aguado, M. J. (Dir.). (2010). Estudio estatal sobre la convivencia escolar en la Educación Secundaria Obligatoria desde las perspectivas del alumnado, el profesorado, los Departamentos de Orientación y los Equipos Directivos. Ministerio de Educación.

Dymnicki, A. B., Weissberg, R. P. \& Henry, D. B. (2011). Understanding how programs work to prevent overt aggressive behaviors: A meta-analysis of mediators of elementary school-based programs. Journal of School Violence, 10(4), 315-337. https://doi.org/10. 1080/15388220.2011.602599

Domeneci, G. (2017). The contribution of new teacher-training to fight bullying and cyberbullying. Journal of Educational, Cultural and Psychological Studies, 16, 11-16.

Engebretson, K. E. (2019). Untangling sexism and classism: attempting complex discussion about sexual violence in teacher education. Gender and Education. https://doi.org/10 $.1080 / 09540253.2019 .1680806$

Elbaz-Luwisch, F. (2009). The uses of conflict in the pedagogy of teacher education. Perspectives in Education, 27(2), 169-176.

Galtung, J. (2003). Paz por medios pacíficos. Paz y conflicto, desarrollo y civilización. Gernika Gogoratuz.

García-Bacete, F. (2009). Formación inicial del profesorado y de los psicopedagogos en educación de la convivencia. Educar, 43, 43-60. https://doi.org/10.5565/rev/educar.121

García-Raga, L. y López-Martín, R. (2011). Convivir en la escuela. Una propuesta para su aprendizaje por competencias. Revista de Educación, 356, 531-555. https://doi.org/104438/1988-592X-RE-2010-356-050 
CARLOS MONGE-LÓPEZ Y PATRICIA GÓMEZ-HERNÁNDEZ EL PAPEL DE LA CONVIVENCIA ESCOLAR EN LA FORMACIÓN INICIAL DEL PROFESORADO DE EDUCACIÓN INFANTIL Y PRIMARIA

Gázquez, J. J., Pérez-Fuentes, M. C. y Carrión, J. J. (2011). Clima escolar y resolución de conflictos según el alumnado: un estudio europeo. Revista de Psicodidáctica, 16(1), 39-58.

Gázquez, J. J., Pérez-Fuentes, M. C., Lucas F. y Fernández, M. (2011). Análisis de la convivencia escolar por los docentes europeos. Aula Abierta, 37(2), 11-18.

Gómez-Jarabo, I. (2015). Situación actual y propuestas sobre la formación del profesorado para la convivencia. Opción, 31(1), 878-984.

Goryl, O., Neilsen-Hewett, C. \& Sweller, N. (2013). Teacher education, teaching experience and bullying policies: Links with early childhood teachers' perceptions and attitudes to bullying. Australian Journal of Early Childhood, 38(2), 32-40. https://doi. org/10.1177/183693911303800205

Grau, R., García-Raga, L. y López-Martín, R. (2017). The challenge of coexistence in socially vulnerable schools. Procedia - Social and Bahavioral Sciences, 237, 710-716. https:// doi.org/10.1016/j.sbspro.2017.02.049

Hastings, T. J., Kavookjian, J., \& Ekong, G. (2019). Associations among student conflict management style and attitudes toward empathy. Currents Pharmacy Teaching and Learning, 11(1), 25-32. https://doi.org/10.1016/j.cptl.2018.09.019

Johnson, A. T. (2019). University infrastructures for peace in Africa: The transformative potential of Higher Education in conflict contexts. Journal of Transformative Education, 17(2), 173-194. https://doi.org/10.1177/1541344618779561

Lázaro-Visa, S. y Fernández-Fuertes, A. (Coords.). (2018). Clima escolar, conflicto y gestión de la convivencia en los centros educativos de Cantabria. Consejería de Educación, Cultura y Deporte.

León, B., Polo, M. I., Gozalo, M. y Mendo, S. (2016). Relevancia del aprendizaje cooperativo sobre los diferentes perfiles de la dinámica bullying. Un análisis mediante pruebas de tamaño del efecto. Anales de Psicología, 32(1), 80-88. https://doi.org/10.6018/analesps.32.1.183141

López, M. C., Tejero, R. y Fernández, A. (2017). Los conflictos escolares y su gestión en la formación inicial del profesorado: un estudio de caso. Profesorado. Revista de Currículum y Formación del Profesorado, 21(4), 294-314.

Luengo, J. A. (2019). El acoso escolar y la convivencia en los centros educativos. Guía para el profesorado y las familias. Consejo Escolar de la Comunidad de Madrid.

Manso, J. (Coord.). (2019). La formación inicial del profesorado en España. Análisis de los planes de estudios tras una década desde su implementación. CNIIE-MEFP. https://sede. educacion.gob.es/publiventa/la-formacion-inicial-del-profesorado-en-espana-analisis-delos-planes-de-estudios-tras-una-decada-desde-su-implementacion/educacion-infantil-yprimaria-educacion-secundaria-formacion-profesores/23106

Mathews, B. (2011). Teacher education to meet the challenges posed by child sexual abuse. Australian Journal of Teacher Education, 36(1), 13-32. http://www.doi.org/10.14221/ ajte.2011v36n1.1

Merma-Molina, G., Ávalos, M. A. y Martínez, M. Á. (2019). ¿Por qué no son eficaces los Planes de Convivencia en España? Revista de Investigación Educativa, 37(2), 561-579. https:// doi.org/10.6018/rie.37.2.313561 
CARLOS MONGE-LÓPEZ Y PATRICIA GÓMEZ-HERNÁNDEZ

EL PAPEL DE LA CONVIVENCIA ESCOLAR EN LA FORMACIÓN INICIAL DEL PROFESORADO DE EDUCACIÓN INFANTIL Y PRIMARIA

Merrett, F. \& Wheldall, K. (2017). Teacher training and classroom discipline. En K. Wheldall (Ed.), Discipline in schools: Psychological perspectives on the Elton Report (2 $2^{\mathrm{a}}$ ed.) (pp. 11-19). Routledge.

Moeyaert, M., Klingbeil, D.A., Rodabaugh, E., \& Turan, M. (en prensa). Three-level metaanalysis of single-case data regarding the effects of peer tutoring on academic and social-behavioral outcomes for at-risk students and students with disabilities. Remedial and Special Education. https://doi.org/10.1177/0741932519855079

Nagle, J. (2018). Twitter, cyber-violence, and the need for a critical social media literacy in teacher education: A review of the literature. Teaching and Teacher Education, 76, 86-94. https://doi.org/10.1016/j.tate.2018.08.014

Negro, A., Mas, C., Monge, C., Martínez, C., Montalvo, D., Lorenzo. E.M., ... y Muñoz, Y. (2017). Formación para la educación inclusiva y mejora de la convivencia. En H. Castillo y G. Nogueiras (Eds.), Nuevas apuestas educativas: entre la docencia presencial y la innovación tecnológica (pp. 147-164). Universidad de Alcalá.

Ochoa, A. C. y Pérez, L. M. (2019). El aprendizaje servicio, una estrategia para impulsar la participación y mejorar la convivencia escolar. Psicoperspectivas. Individuo y Sociedad, 18(1), 1-13. https://doi.org/10.5027/psicoperspectivas-vol18-issue1-fulltext-1478

Peiró, S. (Coord.). (2012). Convivencia en educación: problemas y soluciones. Perspectivas europea y latinoamericana. Universidad de Alicante.

Peñalva, A., Hernández, M. Á. y Guerrero, C. (2013). La gestión eficaz del docente en el aula: un estudio de caso. Revista Electrónica Interuniversitaria de Formación del Profesorado, 16(2), 77-91. https://doi.org/10.6018/reifop.16.2.180931

Pérez, L. M. y Ochoa, A. C. (2017). El aprendizaje-servicio como estrategia para educar en ciudadanía. Alteridad. Revista de Educación, 12(2), 175-187. https://doi.org/10.17163/ alt.v12n2.2017.04

Phillips, D. K. (2010). On transitional space, unresolved conflicts, and an uncertain teacher education. Teachers and Teaching: Theory and Practice, 16(5), 633-644. https://doi.org/ 10.1080/13540602.2010.507970

Reyes, V.C. (2007). Corruption and policy implementation in the Philippines: A comparative analysis of the teacher training and textbook delivery programmes. Asian Journal of Political Science, 15(1), 97-125. https://doi.org/10.1080/02185370701315657

Rodger, S., Bird, R., Hibbert, K., Johnson, A. M., Specht, J. \& Wathen, C. N. (en prensa). Initial teacher education and trauma and violence informed care in the classroom: Results from a preliminary online teacher education course. Psychology in the Schools. https:// doi.org/10.1002/pits.22373

Ryzin, M. J. y Roseth, C. J. (2018). Cooperative learning in middle school: A means to improve peer relations and reduce victimization, bullying, and related outcomes. Journal of Educational Psychology, 110(8), 1192-1201. https://doi.org/10.1037/edu0000265

Sawa, R. J., Barlow, C., Hurlock, D., Irvine, K., Rogers, G. y Myrick, F. (2015). Violence and subjectivity in teacher education. En A.M. Phelan (Ed.), Curriculum theorizing and teacher education: Complicating conjunctions (pp. 41-58). Routledge.

Scholes, L., Jones, C., Stieler-Hunt, C., Rolfe, B., \& Pozzebon, K. (2012). The teachers' role in child sexual abuse prevention programs: Implications for teacher education. Australian Journal of Teacher Education, 37(11), 104-131. http://www.doi.org/10.14221/ajte.2012v37n11.5 
CARLOS MONGE-LÓPEZ Y PATRICIA GÓMEZ-HERNÁNDEZ EL PAPEL DE LA CONVIVENCIA ESCOLAR EN LA FORMACIÓN INICIAL DEL PROFESORADO DE EDUCACIÓN INFANTIL Y PRIMARIA

Silva, E. N. y Rosa, E. C. S. (2013). Professores sabem o que é bullying? Um tema para a formação docente. Psicologia Escolar e Educacional, 17(2), 329-338. https://doi. org/10.1590/S1413-85572013000200015

Teff-Seker, Y. (2020). Peace and conflict in Israeli state-approved textbooks: 2000-2018. Journal of Curriculum Studies, 52(4), 533-550. https://doi.org/10.1080/00220272.2020.1716392

Tirado, R. y Conde, S. (2016). Análisis estructural de la gestión de la convivencia escolar en centros de buenas prácticas de Andalucía (España). Educación XX1, 19(2), 153-178. https://doi.org/10.5944/educxx1.16459

Torrego, J. C. (2006). Desde la mediación de conflictos en centros escolares hacia el modelo integrado de mejora de la convivencia. En J. C. Torrego (Coord.), Modelo integrado de mejora de la convivencia. Estrategias de mediación y tratamiento de conflictos (pp. 11-26). Graó.

Torrego, J. C. (2012). De la investigación a la innovación en mejora de la convivencia y viceversa. En M.T. Castilla y V.M. Martín (Coords.), Buenas prácticas en educación intercultural y mejora de la convivencia (pp. 285-300). Wolters Kluwer.

Torrego, J. C., Bueno, Á., Caballero-García, P. Á., Parra, J., Gomariz, M. Á., ... y Pedrajas, M. L. (2019). La participación en los centros educativos de la Comunidad de Madrid. La participación de las familias y el alumnado. Consejo Escolar de la Comunidad de Madrid. http://www.madrid.org/bvirtual/BVCM016449.pdf

Toshalis, E. (2010). From disciplined to disciplinarian: The reproduction of symbolic violence in pre-service teacher education. Journal of Curriculum Studies, 42(2), 183-213. https:// doi.org/10.1080/00220270903267972

Turk, F. (2018). Evaluation of the effects of conflict resolution, peace education and peer mediation: A meta-analysis study. International Education Studies, 11(1), 25-43. https:// doi.org/10.5539/ies.v11n1p25

Uruñuela, P. M. (2018). Trabajar la convivencia en los centros educativos. Una mirada al bosque de la convivencia ( $3^{\mathrm{a}}$ ed.). Narcea Ediciones.

Vega, M. A. (2017). Convivencia escolar: dimensiones, programas y evaluación. Universidad Pública de Navarra. http://www.unavarra.es/clinical-psychology/tesis-doctorales?index=2

Verdeja, M. (2012). Aprendizaje de la convivencia en contextos educativos: identificando problemas y formulando propuestas de mejora desde la acción tutorial. Revista Latinoamericana de Educación Inclusiva, 6(2), 167-190. http://rinace.net/rlei/numeros/ vol6-num2/art7.pdf

Zabalza, M. Á. (2002). Situación de la convivencia escolar en España: políticas de intervención. Revista Interuniversitaria de Formación del Profesorado, 44, 139-174. https://redined.mecd. gob.es/xmlui/bitstream/handle/11162/32215/014200230421.pdf?sequence=1\&isAllowed=y

Zaitegi, N. (2010). La educación en y para la convivencia positiva en España. Revista Iberoamericana sobre Calidad, Eficacia y Cambio en Educación, 8(2), 93-132. https://revistas. uam.es/reice/article/view/5362/5801 